\title{
Silencing TRPC1 expression inhibits invasion of CNE2 nasopharyngeal tumor cells
}

\author{
BENFU HE ${ }^{1,2^{*}}$, FEIYE LIU $^{1 *}$, JIAN RUAN $^{1 *}$, AIMIN LI $^{1}$, JINZHANG CHEN $^{1}$, \\ RONG LI $^{1}$, JIE SHEN ${ }^{1}$, DAYONG ZHENG ${ }^{1}$ and RONGCHENG LUO ${ }^{1}$ \\ ${ }^{1}$ Department of Oncology, Nanfang Hospital, Southern Medical University, Guangzhou 510515; \\ ${ }^{2}$ Department of Oncology, 421 Hospital of PLA, Guangzhou 510318, P.R. China
}

Received December 6, 2011; Accepted January 9, 2012

DOI: $10.3892 / 0 r .2012 .1695$

\begin{abstract}
The invasion and metastasis processes involved in nasopharyngeal carcinoma (NPC) remain enigmatic. Transient receptor potential channel-related protein 1 (TRPC1) is a cation channel involved in diverse cellular functions by precisely controlling $\mathrm{Ca}^{2+}$. The role of this unique TRPC member in nasopharyngeal malignancies has not yet been delineated. Here, we downregulated TRPC1 in CNE2 cells by RNAi technology and by using 2-APB, an inhibitor of the inositol 1,4,5-trisphosphate $\left(\mathrm{IP}_{3}\right)$ receptor and of store-operated $\mathrm{Ca}^{2+}$ channel-mediated $\mathrm{Ca}^{2+}$ entry. Both types of TRPC1 inhibition resulted in significantly attenuated adhesive and invasive abilities, suggesting that TRPC1 can modulate the metastasis of NPC. These findings support further investigation of the potential of TRPC1 as a novel therapeutic target for intervention in nasopharyngeal carcinoma.
\end{abstract}

\section{Introduction}

Nasopharyngeal carcinoma (NPC) is a distinct disease that differs in epidemiology, histopathology, clinical characteristics and therapy from other cancers. Although the survival rates of early diagnosed patients has been improved due to the prevalence of new detection and treatment methods, the long-term survival advantage of nasopharyngeal carcinoma patients has not yet been significantly prolonged $(1,2)$. Oncogenesis of NPC has been proven to be a multiple-step and coherent process that involves many aberrant alterations

Correspondence to: Dr Dayong Zheng or Dr Rongcheng Luo, Department of Oncology, Nanfang Hospital, Southern Medical University, Guangzhou 510515, Guangdong Province, P.R. China E-mail: zhengdayong@gmail.com

E-mail: luorc0001@126.com

${ }^{*}$ Contributed equally

Key words: transient receptor potential channel, transient receptor potential channel-related protein 1 , nasopharyngeal carcinoma, invasion of key signaling cascades, more specifically, various mutations of proteins encoded by oncogenes and tumor suppressor genes as well as modulations of several other gene products (3). However, the invasion and metastasis process involved remains enigmatic. Thus, elucidating the mechanism underlying the metastasis of NPC is still urgently needed for a better individualized target therapy.

Calcium $\left(\mathrm{Ca}^{2+}\right)$ regulates physiological and pathological processes ubiquitously by modulating relevant signaling pathways and proteins. However, unique biological events may activate or inhibit different channels $(4,5)$. The transient receptor potential channel (TRP) is a superfamily of cation channel that participates in certain physiological and pathological processes by modulating amplitude and temporal-spatial aspects of the intracellular $\mathrm{Ca}^{2+}$ currents, consisting of six main subfamilies termed TRPC, TRPV, TRPM, TRPP, TRPML and TRPA $(6,7)$. A plethora of evidence has proven that the TRPC family is involved in the development of diverse malignancies. TRPC 3 can promote the cell growth and tumor formation of ovarian cancer (8), while TRPC6 stimulates cellular proliferation of glioma, prostate cancer and hepatoma cells (9-11). However, only a few studies have investigated the role of TPRC channels in the progression of cancer. As a member of the TRP canonical-subfamily, transient receptor potential channel-related protein 1 (TRPC1) is also a component of a store-operated $\mathrm{Ca}^{2+}$ channel (SOCs) that can be activated by depletion of the internal $\mathrm{Ca}^{2+}$ from the cytoplasmic storage compartments and tightly control $\mathrm{Ca}^{2+}$ homeostasis by forming homomultimers or heteromultimers with STIM1, Orai1 and other TRPC members (12-17). It has been shown that TRPC1 is expressed in various kinds of tumor cells, including prostate cancer cells $(18,19)$ and human breast cancer cells (20). Our preliminary studies have noted elevated levels of TRPC1 expression in tissues and cell lines of NPC as compared to those of normal or other nasopharyngeal disease origin (unpublished data), which lead us to speculate that, as a separately classified subgroup of TRPCs, TRPC1 also influences the development of nasopharyngeal malignancies.

In this study, we attempt to explore the possible functions of TRPC1 in the CNE2 NPC cell line by observing changes of cellular biological behaviors following downregulation of TRPC1 in CNE2 with RNAi technique and the inhibitor, 2-APB. 
Table I. Oligonucleotide sequences of TRPC1-specific shRNAs.

shRNA1

Sense 5'-GATCCCCGCATTCCAGGTTTCGTCTTGATTCAAGAGATCAAGACGAAACCTGGAATGCTTTTTA-3' $658-678$ Antisense 5'-AGCTTAAAAAGCATTCCAGGTTTCGTCTTGATCTCTTGAATCAAGACGAAACCTGGAATGC GGG-3' ShRNA2

Sense 5'-GATCCCCGCTCTATCTTGGGTCCATTACTTCAAGAGAGTAATGGACCCAAGATAGAGCTTTTTA-3' Antisense 5'-AGCTTAAAAACTCTATCTTGGGTCCATTACTCTCTTGAAGTAATGGACCCAAGATAGAGCGG-3' ShRNA3

Sense 5'-GATCCCCGGCAAGGTCAAACGGCAAA TTCAAGAGATTTGCCGTTTGACCTTGCCTTTTTA-3' Antisense 5'-AGCTTAAAAA GGCAAGGTCAAACGGCAAA TCTCTTGAATTTGCCGTTTGACCTTGCCGGG-3'

Negative control

Sense 5'-GATCCCCGCCAGCTTAGCACTGACTCTTCAAGAGAGAGTCAGTGCTAAGCTGGCTTTTTA-3' Antisense 5'-AGCTTAAAAAGCCAGCTTAGCACTGACTCTCTCTTGAAGAGTCAGTGCTAAGCTGGCGGG-3'

\section{Materials and methods}

Cell culture and reagent. The C666-1, 6-10B and CNE2 cell lines derived from human nasopharyngeal carcinomas and 293FT cells with various degrees of metastatic behavior were cultured in RPMI-1640 supplemented with $10 \%$ fetal bovine serum (Gibco, Paisley, UK) at $37^{\circ} \mathrm{C}$ in a humidified $5 \% \mathrm{CO}_{2}$-air atmosphere, $100 \mathrm{U} / \mathrm{ml}$ penicillin, and $100 \mu \mathrm{g} / \mathrm{ml}$ streptomycin. 2-APB, a well-known inhibitor of the IP3 receptor and of the SOCs-mediated $\mathrm{Ca}^{2+}$ entry in the cells was purchased from Santa Cruz Biotechology, Inc.

$q R T-P C R$. Total-RNA of three nasopharyngeal carcinoma cell lines was extracted using the TRIzol reagent according to the manufacturer's instructions (Invitrogen Life Technologies). Two micrograms total-RNA was transcribed to complementary DNA (cDNA) in a $20 \mu 1$ reaction using an access reverse transcription system (Takara Bio, Inc., Japan). The quantitative PCR primer sequences of TRPC1 (forward, 5'-CCGGCAT TCCAGGTTTCGT-3' and reverse, 5'-TCCACCTCCACAA GACTTAG-3') were used. The quantitative RT-PCR reaction was carried out using a commercial SYBR-Green reaction mix (Takara Bio, Inc.). Thermal cycling was performed using a MJ Chromo4 real-time RT-PCR thermocycler (Bio-Rad, Hercules, $\mathrm{CA}$ ), and the quantitative PCR conditions were as follow: $95^{\circ} \mathrm{C}$ for $2 \mathrm{~min}$ and 40 cycles at $95^{\circ} \mathrm{C}$ for $30 \mathrm{sec}$, followed by $60^{\circ} \mathrm{C}$ for $35 \mathrm{sec}$. Experiments were performed in triplicate in the same reaction. CNE2 cells were found to significantly express more TRPC1 than the other cell lines and were thus chosen for further investigation.

Chemical treatment, shRNA preparation, plasmids construction and transfection. CNE2 was cultured under control condition and 2-APB $(75 \mu \mathrm{mol} / 1)$ to investigate the effect of TRPC1 inhibition. Four pairs of shRNA were designed according to the TRPC1 sequence in GenBank (NM_003304) to verify the specific effect of TRPC1 on the invasion of NPC. As shown in Table I, DNA oligonucleotides targeting TRPC1 were synthesized and inserted into BglII-HindIII linear- ized pSUPERretro-puro-TRPC1-shRNA expression vector according to manufacturer's instructions. All the inserted sequences were verified by DNA sequencing. Each vector contains the puromycin-resistance gene to provide puromycin resistance in mammalian cells. Efficiency of interference was evaluated by qRT-PCR as described before and by western blot analysis. The chosen constructed lentiviral plasmids were hereafter denoted as pSUPERretro-puro-TRPC1-shRNA1 for specific interfering of CNE2 and pSUPERretro-purovector for the negative control. Lentiviruses were generated in 293FT cells by co-transfection of pSUPERretro-puroTRPC1-shRNA1 or pSUPERretro-puro-vector, with PIK plasmids. Then lentiviral particles were harvested from the media $48 \mathrm{~h}$ after transfection, purified with ultracentrifugation and interfered with CNE2. Stable cell lines were selected with mediums containing $0.5 \mu \mathrm{g} / \mathrm{ml}$ puromycin, named CNE2-shTRPC1 and CNE2-CON. TRPC1, MMP2 and MMP9 expression of each group were detected with QRT-PCR and immunocytochemistry. The primers of MMP2, were forward, 5'-AGGCCAAGTGGTCCGTGTGA-3' and reverse, 5'-TAGGTGGTGGAGCACCAGAG-3'; MMP9, forward, 5'-TCTGGAGGTTCGACGTGAAG-3' and reverse, 5'-TTGGTCCACCTGGTTCAACT-3'; and for $\beta$-actin, forward, 5'-TGGCACCCAGCACAATGAA-3, reverse 5'-CTAAGTCATAGTCCGCCTAGAAGCA-3'.

Western blotting. Cells were lysed with lysis buffer (PBS-1\% Triton X-100) containing protease inhibitors and centrifuged at $15,000 \mathrm{rpm}$ for $15 \mathrm{~min}$ at $4^{\circ} \mathrm{C}$ to remove insoluble materials. The resulting supernatants were collected and their protein concentration was determined by the BCA method. Proteins were resolved by SDS-PAGE, transferred to PVDF membranes using the 'Semidry-transfer method' (Bio-Rad), and analyzed by western blotting using anti-TRPC1, antiGAPDH or anti- $\beta$-actin primary antibodies (Santa Cruz Biotechology, Inc.). Then membranes were incubated with corresponding secondary horseradish peroxidase-conjugated antibodies. Protein bands were detected using the ECL reagent (Amersham Biosciences). 
Transwell migration assay. The migratory ability of transfected cells was determined by their ability to cross the $8-\mu \mathrm{m}$ pores of migration chambers (Corning, Acton, MA). In brief, $1.0 \times 10^{5}$ cells $/ 200 \mu 1$ serum-free medium were plated in the upper transwell chambers, whereas medium with $10 \%$ fetal bovine serum was added to the lower well. After incubating for $24 \mathrm{~h}$, the cells on the upper side of the inserts were removed by cotton swab. The inserts were fixed in methanol and stained with hematoxylin and eosin. The number of migrated cells attached to the other side of the insert was counted under a light microscope in five random fields at a magnification of x200. Numbers of migrated cells under different treatments were normalized to the control.

Transwell invasion assay. Transwell invasion assays were carried out essentially as described above for the migration assays, but in the presence of ECMatrix (Chemicon).

Immunocytochemistry. The transfected cells were grown on glass slides, fixed with acetone, and endogenous peroxidase was blocked by incubation with $0.3 \% \mathrm{H}_{2} \mathrm{O}_{2}$ in methanol. The sections were washed and incubated overnight with a 1:300, 1:100, 1:150 dilution of anti-TRPC1, MMP2, MMP9 antibody (Santa Cruz Biotechology, Inc.) at $4^{\circ} \mathrm{C}$, respectively. After subsequent washings in PBS, the secondary antibody was added and incubated for $1 \mathrm{~h}$ at room temperature. After another wash in PBS, the peroxidase activity was localized by staining with diaminobenzidine as the substrate, and sections were rinsed in water, dried and mounted.

Statistical analysis. SPSS 13.0 software (Abbott Laboratories, North Chicago, IL) was used for statistical analysis. Results of qRT-PCR, western blot analysis and the Transwell assay were assessed using one-way ANOVA. Differences were considered statistically significant when $\mathrm{P}<0.05$.

\section{Results}

Expression of TRPC1 in three nasopharyngeal carcinoma cell lines. Due to our previous finding that TRPC1 expression was significantly higher in NPC cells and tissues, we compared TRPC1 expression of three NPC cell lines using qRT-PCR. The results showed that the CNE2 cells with metastatic ability expressed elevated levels of TRPC1 mRNA (1) as compared with the 6-10B $(0.662 \pm 0.037)$ and C666-1 $(0.736 \pm 0.096)$ cells $(\mathrm{P}<0.05$ for both) (Fig. 1).

Vector stably expressing TRPCl shRNA as well as 2-APB downregulated the expression of TRPCl. The knock-down efficiencies of different specific shRNAs in CNE2 cells were first evaluated using qRT-PCR and western blot analysis. shRNA1 showed the most remarkable decrease in TRPC1 mRNA $(0.345 \pm 0.047)$ and protein $(0.182 \pm 0.191)$ expression and then were chosen for further interference of CNE2 (Fig. 2). Stable cell lines named CNE2-shTRPC1 and CNE2-CON were established after transfection and selective culture. CNE2 treated with 2-APB was named CNE2-2-APB. TRPC1 expression of each group was evaluated by qPCR and western blotting. Relative TPPC1 mRNA levels in individual cell lines were normalized against mRNA levels of an internal

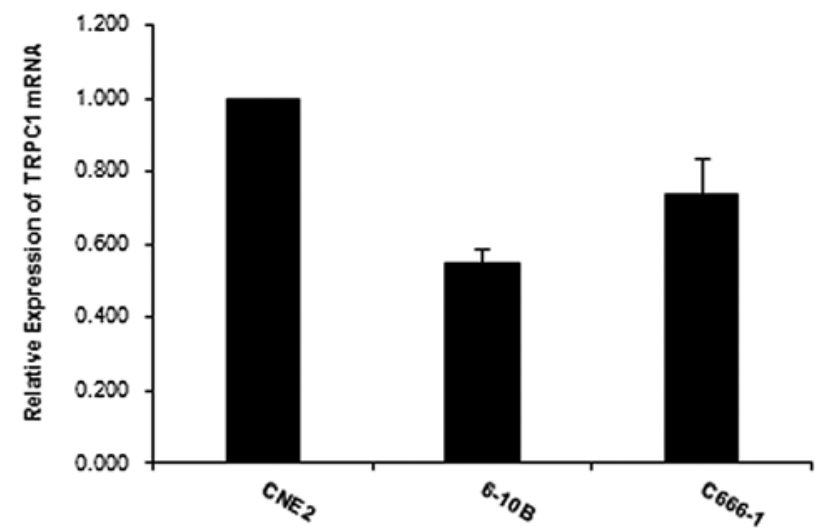

Figure 1. TRPC1 expression in three human NPC cell lines. Quantification of TRPC1 mRNA expression in different cells related to controls as detected by qRT-PCR. CNE2 expressed the highest level of TRPC1 mRNA. The expression of $\beta$-actin protein and mRNA was also examined and served as controls for sample loading. The figure shows a representative result of three independent experiments.

A

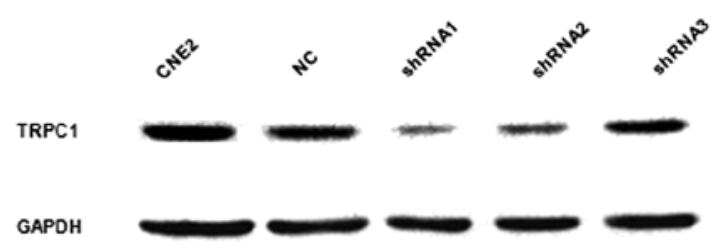

B

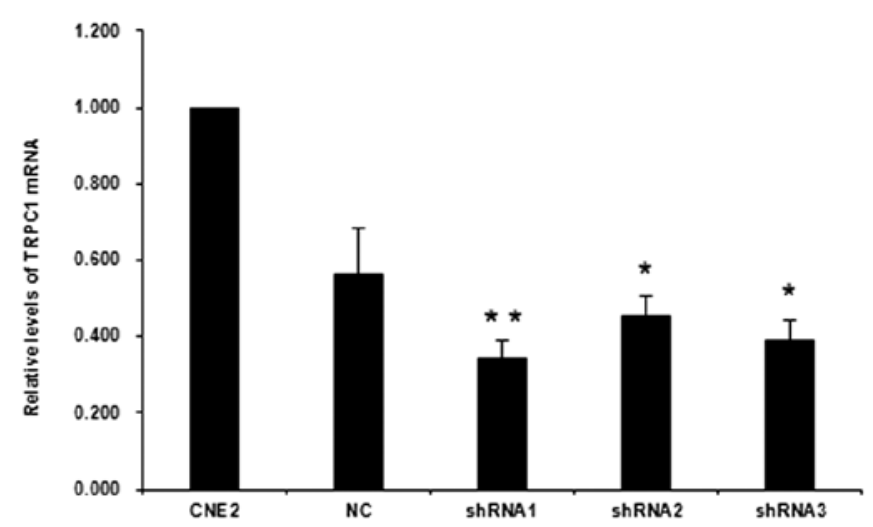

Figure 2. TRPC1 specific shRNA results in the reduction of TRPC1 mRNA and protein in CNE2 cells. (A) The efficiencies TRPC1 shRNA1-3 were detected by qRT-PCR and western blotting, negative-targeting shRNA was set as control in both experiments. (B) The levels of TRPC1 mRNA and protein in stable established cell lines were assessed by qRT-PCR and western blotting, respectively. Both quantitative analyses of the mRNA and protein levels of TRPC1 normalized to $\beta$-actin and GAPDH, respectively. Data were mean \pm SE of three independent experiments. $\left({ }^{*} \mathrm{P}<0.05\right.$ and ${ }^{* *} \mathrm{P}<0.01$ as compared to $\mathrm{CNE} 2$ ).

control gene, $\beta$-actin, performed in the same run. The CNE2shTRPC1 $(0.147 \pm 0.018)$ and CNE2-2-APB $(0.258 \pm 0.033)$ showed significantly reduced transcriptions of TRPC1 mRNA as comparing with either CNE2 (1) or CNE2-CON $(0.994 \pm 0.049)$, respectively $(\mathrm{P}<0.05)$ (Fig. 3A). Similarly, western blot analysis demonstrated a significantly declined level of TRPC1 protein in CNE2-shTRPC1 $(1.98 \pm 0.096)$ and 
A

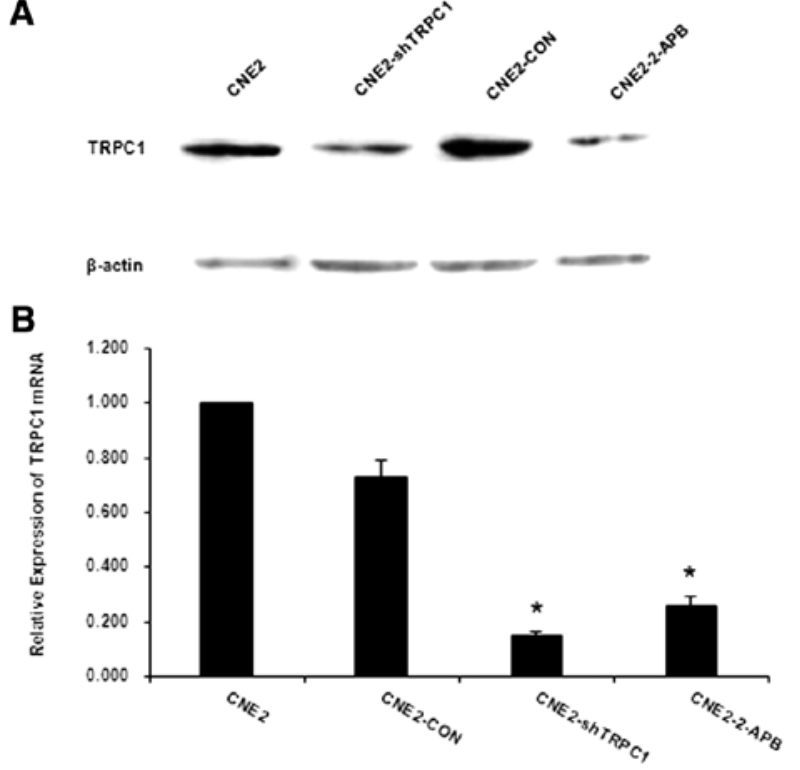

Figure 3. 2-APB causes effective downregulation of TRPC1 expression. (A) The levels of TRPC1 protein were assessed by western blotting. The results revealed that protein production of TRPC1 in CNE2 cells was both effectively inhibited with CNE2-shTRPC1 interference and the nonspecific TRPC1 channel inhibitor 2-APB. (B) Levels of $\beta$-actin were evaluated as an internal control for loading, compared with CNE2 or CNE2-CON. The expression levels of TRPC1 protein in CNE2-shTRPC1 and CNE2-2-APB were significantly. decreased (" $\mathrm{P}<0.05$ vs. CNE2).

A
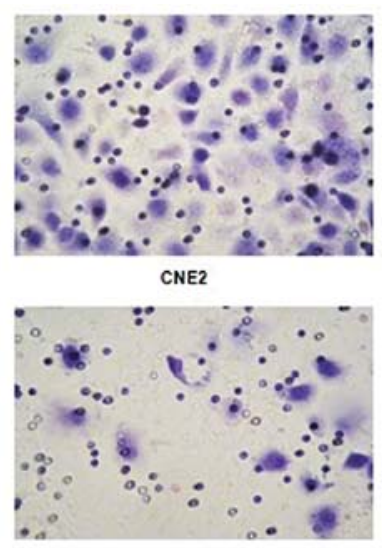

CNE2-ShTRPC1

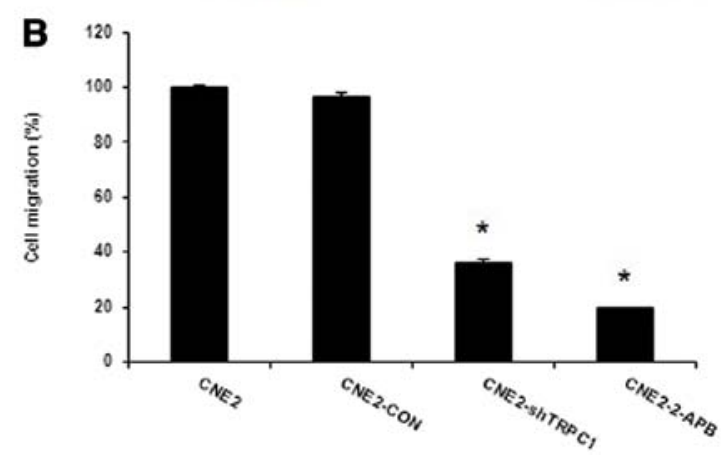

Figure 4. Inhibition of the TRPC7 channel and knockdown of TRPC1 suppress cancer migration. (A) The transwell migration system demonstrates that inhibition of the TRPC7 channel and knockdown of TRPC1 causes reduction of migrated cell numbers in CNE2 cells. (B) Quantitative Transwell migration assay. The Y-axis represents the number of migrated cells. The data were the average numbers of cells that migrated in a representative experiment, measured in triplicate and presented as mean $\pm \mathrm{SD}$; Magnification, $\mathrm{x} 200$. ( ${ }^{*} \mathrm{P}<0.05$ as compared to CNE2).
A
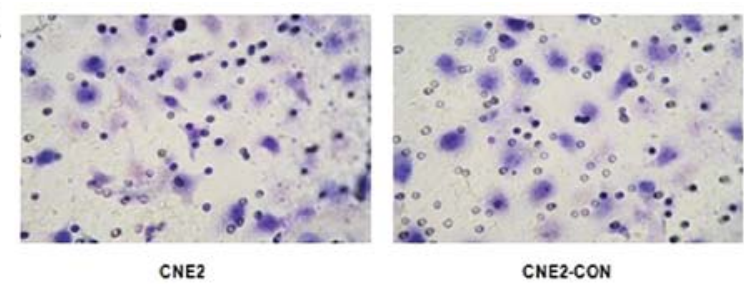

CNE2-CON

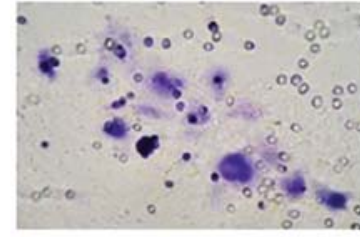

CNE2-ShTRPC1

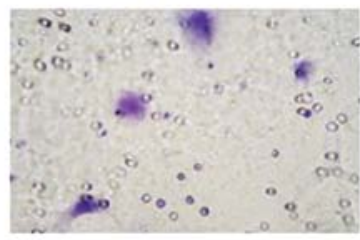

CNE2-2-APB

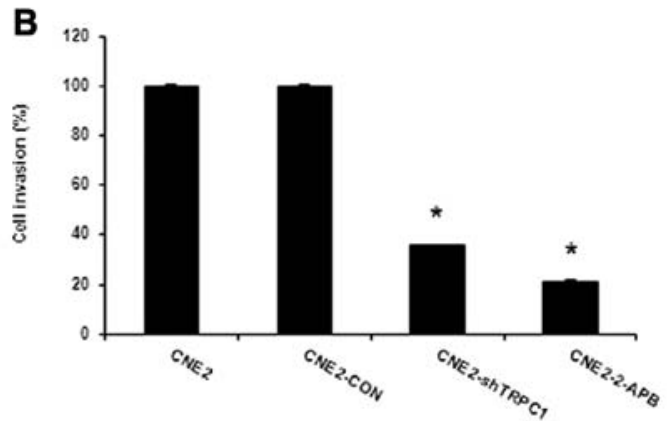

Figure 5. Cell invasion capability was detected by a transwell assay in CNE2, CNE2-CON, CNE2-shTRPC1 and CNE2-2-APB. (A) TRPC1-deficient cells showed reduction of migrated cell numbers, moreover, the addition of 2-APB also reduced the migration of CNE2 cells, indicating impaired invasive ability. (B) Quantitative Transwell invasion assay. The Y-axis represents the number of invasive cells. The data were the average numbers of cells that migrated in a representative experiment, measured in triplicate and presented as the mean \pm SD. Magnification, $\mathrm{x} 200$ ( $\mathrm{P}<0.05$ vs. CNE2).

CNE2-2-APB (1.81 \pm 0.09$)$ as compared with either CNE2 $(3.88 \pm 0.183)$ or $\mathrm{CNE} 2-\mathrm{CON}(3.77 \pm 0.436)(\mathrm{P}<0.05)$ (Fig. 3B).

Effects of TRPC1 downregulation on cell migration activity. CNE2-shTRPC1 and CNE2-2-APB displayed remarkable diminishments in migration ability as compared with either CNE2-CON or CNE2 ( $\mathrm{P}<0.05$, for both). The migration rates of CNE2-shTRPC1 and CNE2-2-APB were 36.5 and $19.6 \%$, while those of the CNE2 and CNE2-CON groups were 100 and $96.9 \%$, respectively (Fig. 4). Inhibition of TRPC1 cause significantly attenuated migration of CNE2 cells.

Effects of TRPC1 downregulation on cell invasion activity. Invasion assays were carried out using Matrigel-coated Transwell culture chambers. After $24 \mathrm{~h}$ invading cells were counted using image analysis. CNE2-shTRPC1 and CNE22-APB showed much lower invasion activities than either CNE2-CON or CNE2 (P $<0.05$, respectively) (Fig. 5). The invasion rates of the CNE2, CNE2-CON, CNE2-shTRPC1 and CNE2-2-APB groups were 100, 99.7, 35.6 and 21.4\%, respectively. Suppression of TRPC1 led to a significant decrease in the invasion of CNE2 cells.

Effects of TRPC1 inhibition on MMP2, MMP9 expression. Immunocytochemistry and qRT-PCR were employed to detect 
A

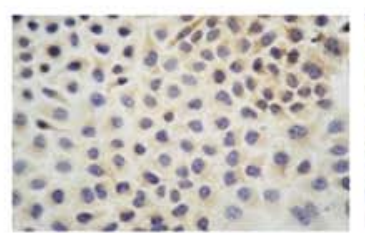

CNE2 MMP2

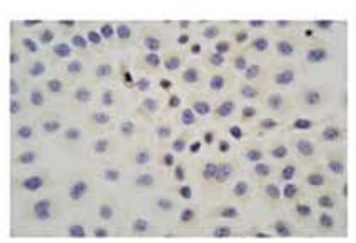

CNE2-ShTRPC1 MMP2

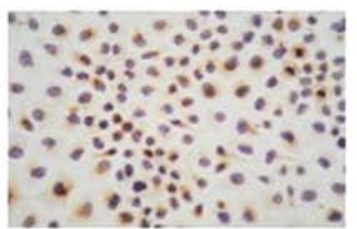

CNE2 MMP9

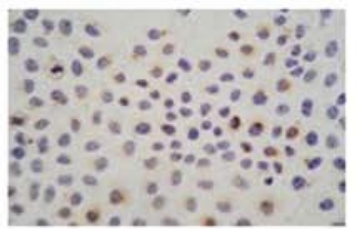

CNE2-ShTRPC1 MMP9

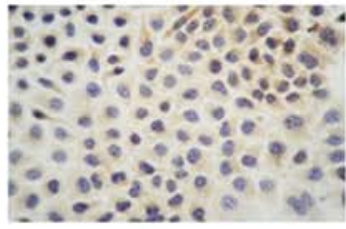

CNE2-CON MMP2

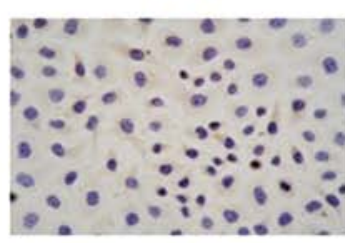

CNE2-2-APB MMP2

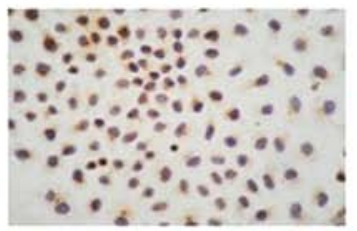

CNE2-CON MMPS

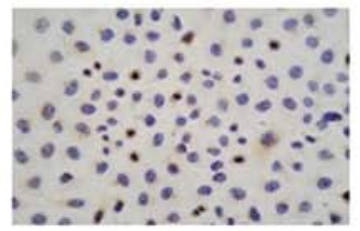

CNE2-2-APB MMPS
B
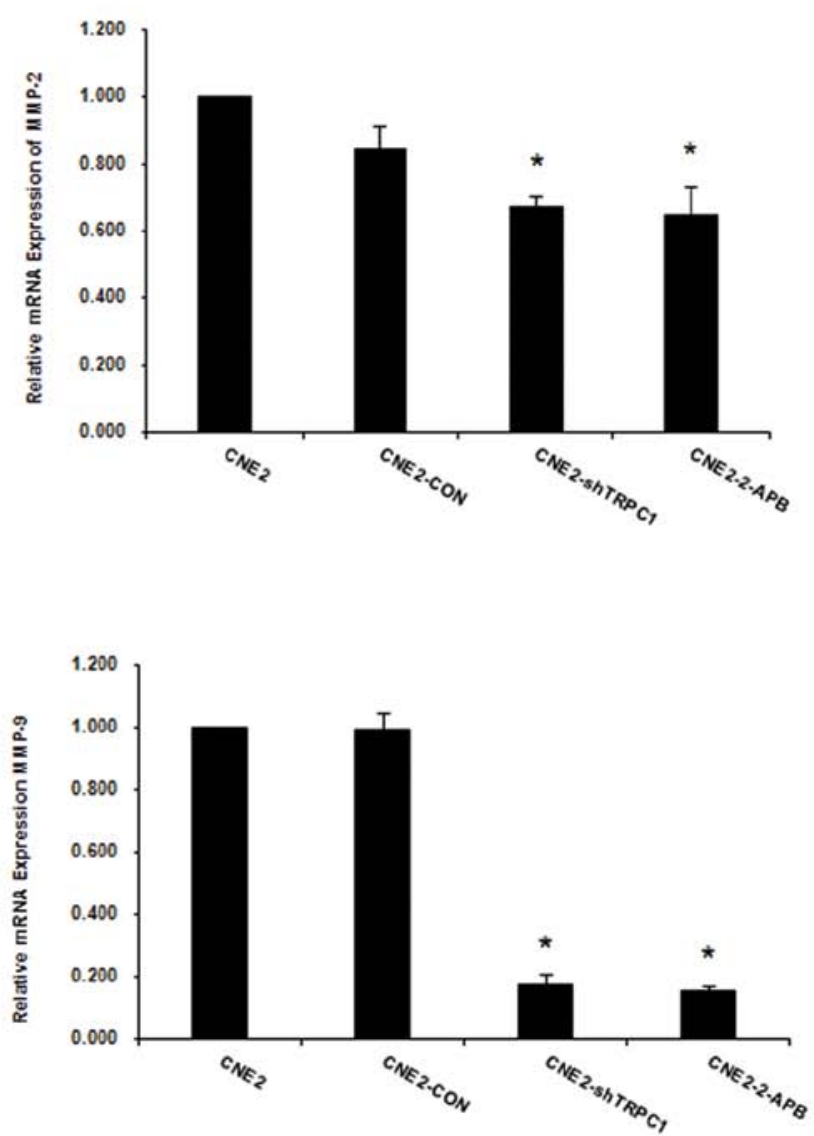

Figure 6. MMP2 and MMP9 expression in CNE2, CNE2-CON, CNE2-shTRPC1 and CNE2-2-APB. (A) Immunocytochemistry analysis showed reduced levels of MMP2 and MMP9 protein in CNE2-shTRPC1 cells and CNE2-2-APB. (B) The qRT-PCR analysis also demonstrated that MMP2 and MMP9 were both expressed at the lowest levels in CNE2-shTRPC1 cells and CNE2-2-APB in comparison with the control ("P<0.05 and ${ }^{* *} \mathrm{P}<0.01$ as compared to CNE2).

the expression of MMP2 and MMP9 in CNE2-shTRPC1, CNE2-2-APB, CNE2-CON and CNE2. A strong staining pattern of MMP2 and MMP9 was seen in the cytoplasm of CNE2-CON and CNE2, while a much weaker pattern of MMP2 and MMP9 was observed in the cytoplasm of CNE2shTRPC1 and CNE2-2-APB (Fig. 6A).

Meanwhile, inhibition of TRPC1 resulted in significant diminishments of MMP2 and MMP9 expression as measured by qRT-PCR. The resulted also demonstrated that the mRNA levels of MMP2 and MMP9 in CNE2-shTRPC1 and CNE22 -APB were lower than both control groups (ratio to $\beta$-actin being $0.671 \pm 0.030 \%$ and $0.175 \pm 0.029 \%$ in CNE2-shTRPC1, $0.650 \pm 0.085 \%$ and $0.154 \pm 0.0164 \%$ compared with negative control groups ( $\mathrm{P}<0.05$ for both) (Fig. 6B).

\section{Discussion}

TRPC1 is a mammalian homologue of Drosophila TRP as well as a core component of store-operated calcium channels (SOCs) which mediate store-operated calcium entry (SOCE) and thus delicately tune cellular $\mathrm{Ca}^{2+}$ levels. Since cytoplasmic $\mathrm{Ca}^{2+}$ has an impact on numerous crucial cellular functions relevant to tumorigenesis and progression, like cell proliferation, apoptosis, differentiation and angiogenesis $(4,21)$, it is not unexpected to find that TRPC1 was involved in the development of various cancers. In this study, we found that inhibition of TRPC1 channels by either RNA interference or 2-APB not only leads to significant attenuated migratory and invasive abilities of an NPC cell line CNE2 but is also accompanied by notable increases of MMP2 and MMP9 expression, indicating a crucial role of $\left(\mathrm{Ca}^{2+}\right)_{\mathrm{i}}$ regulated by TRPC1 in the invasion and metastasis of NPC.

Previous data have proven that TRPC1 is of great importance for cellular polarity and migration directing in neuritis, neutrophils and transformed renal epithelial cells (22-24). In addition to the above studies, we found that TRPC1 are also implicated in the migration of NPC cells. Cellular movement is an essential step for the invasion of malignant cells. $\mathrm{Ca}^{2+}$ channels can finely tune morphological polarities and factors contributing to the migration of various cells including those of tumor origins by controlling $\mathrm{Ca}^{2+}$ homeostasis (24). Since $\mathrm{Ca}^{2+}$ signals in different cells are regulated highly spatially and temporally by specific channels, our results that inhibition of TRPC1 by either RNA interference or 2-APB abates the migration of CNE2, indicates that migration ability of nasopharyngeal carcinoma cells correlates with TRPC1 expression and that TRPC1 silencing alone is sufficient to attenuate the migration of nasopharyngeal carcinoma cells. TRPC1 may 
play a role in the metastasis of CNE2 through stimulation of migration of malignant cells.

Results of the Transwell assay showed an association between the attenuated expression of TRPC1 and impairment of the invasive ability in CNE2. $\mathrm{Ca}^{2+}$ signaling has been implicated in the invasion and metastasis of tumors, such as lung cancer, fibrosarcoma and prostate cancer through various channels (25-27). Alternation of $\mathrm{Ca}^{2+}$ depends on various channel ATPases and exchangers which are uniquely distributed in different cells and tissues. TRPC1, as an important component of $\mathrm{Ca}^{2+}$ regulating channels, may play a crucial role in the progression of NPC. Since CNE2 is an NPC cell line with a relatively high invasive ability compared to the 6-10F and C666-1 cells, the finding that mRNA and protein levels of TRPC1 were significantly elevated in CNE2 also support a relation between the functional expression of TRPC1 and invasion of NPC.

Further, we detected a downregulation of MMP2 and MMP9 in CNE2 cells with the silencing of TRPC1. As type IV collagenases, MMP2 and MMP9 have been previously demonstrated to facilitate both formation and progression of different malignancies by degrading the extracellular matrix (28-30). Besides, MMP2 and MMP9 are independent prognostic factors for NPC patients and elevated levels of their expression have been correlated with poor outcomes of patients $(31,32)$. As the expression of MMP2 and MMP9 decreased significantly following TRPC1 downregulation in CNE2 cells, the stimulatory effects of TRPC1 on the invasion of CNE2 may be not only consequences of the enhanced cellular migration, but also related to the proteolytic mechanism of MMP2 and MMP9.

Our study also finds that 2-APB can inhibit both expression and function of TRPC1.2-APB, an inhibitor of the inositol 1,4,5-trisphosphate $\left(\mathrm{IP}_{3}\right)$ receptor, has been reported to exert different effects on TRP channels (33). Certain TRPC channels (TRPC1, TRPC3, TRPC5 and TRPC6) and TRPM channels (TRPM3, TRPM7 and TRPM8) can be suppressed by 2 -APB, while some TRPV channels (TRPV1, TRPV2 and TRPV3) can be stimulated by 2 -APB. In accordance with previous findings that 2 -APB can nonspecifically block TRPC1 at $75 \mu \mathrm{mol} / 1$ (34), we here demonstrated that $75 \mu \mathrm{mol} / 12$-APB can significantly suppress the expression and function of TRPC1 in CNE2.

In conclusion, our study demonstrates that inhibition of TRPC1 channels could suppress cellular motility and invasion of CNE2, which was accompanied with a simultaneous MMP2 and MMP9 decrease $(32,33)$. The results that RNA interference and inhibition via 2-APB yield similar results in terms of TRPC1 expression or the invasive abilities of CNE2 indicates an important role of TRPC1 in the invasion of NPC. Further investigations to delineate the specific molecular mechanisms are worthy of extensive attention. TRPC1 may be a potent therapeutic target for NPC treatment.

\section{Acknowledgements}

We gratefully thank Mr. Chengwei Lv from The Cancer Biotherapy Center of Nanfang Hospital (Guangzhou, China) for the collection and maintenance of the NPC cell lines used in this study and excellent technical assistance.

\section{References}

1. Vokes EE,Liebowitz DN and WeichselbaumRR: Nasopharyngeal carcinoma. Lancet 350: 1087-1091, 1997.

2. Yu MC and Yuan JM: Epidemiology of nasopharyngeal carcinoma. Semin Cancer Biol 12: 421-429, 2002.

3. Molinolo AA, Amornphimoltham P, Squarize $\mathrm{CH}$, Castilho RM, Patel V and Gutkind JS: Dysregulated molecular networks in head and neck carcinogenesis. Oral Oncol 45: 324-334, 2009.

4. Berridge MJ, Bootman MD and Roderick HL: Calcium signalling: dynamics, homeostasis and remodelling. Nat Rev Mol Cell Biol 4: 517-529, 2003.

5. Berridge MJ, Lipp P and Bootman MD: The versatility and universality of calcium signalling. Nat Rev Mol Cell Biol 1: 11-21, 2000.

6. Nilius B, Voets T and Peters J: TRP channels in disease. Sci STKE 2005: e8, 2005 .

7. Clapham DE, Runnels LW and Strubing C: The TRP ion channel family. Nat Rev Neurosci 2: 387-396, 2001.

8. Yang SL, Cao Q, Zhou KC, Feng YJ and Wang YZ: Transient receptor potential channel $\mathrm{C} 3$ contributes to the progression of human ovarian cancer. Oncogene 28: 1320-1328, 2009.

9. Thebault S, Flourakis M and Vanoverberghe K, et al: Differential role of transient receptor potential channels in $\mathrm{Ca}^{2+}$ entry and proliferation of prostate cancer epithelial cells. Cancer Res 66: 2038-2047, 2006.

10. El BC, Bidaux G, Enfissi A, Delcourt P, Prevarskaya N and Capiod T: Capacitative calcium entry and transient receptor potential canonical 6 expression control human hepatoma cell proliferation. Hepatology 47: 2068-2077, 2008.

11. Ding X, He Z and Zhou K, et al: Essential role of TRPC6 channels in $\mathrm{G} 2 / \mathrm{M}$ phase transition and development of human glioma. J Natl Cancer Inst 102: 1052-1068, 2010.

12. Wes PD, Chevesich J, Jeromin A, Rosenberg C, Stetten G and Montell C: TRPC1, a human homolog of a Drosophila storeoperated channel. Proc Natl Acad Sci USA 92: 9652-9656, 1995.

13. Ambudkar IS: TRPC1: a core component of store-operated calcium channels. Biochem Soc Trans 35: 96-100, 2007.

14. Strubing C, Krapivinsky G, Krapivinsky L and Clapham DE: Formation of novel TRPC channels by complex subunit interactions in embryonic brain. J Biol Chem 278: 39014-39019, 2003.

15. Zagranichnaya TK, Wu X and Villereal ML: Endogenous TRPC1, TRPC3, and TRPC7 proteins combine to form native store-operated channels in HEK-293 cells. J Biol Chem 280: 29559-29569, 2005.

16. Ambudkar IS, Ong HL, Liu X, Bandyopadhyay BC and Cheng KT: TRPC1: the link between functionally distinct storeoperated calcium channels. Cell Calcium 42: 213-223, 2007.

17. Yuan JP, Zeng W, Huang GN, Worley PF and Muallem S: STIM1 heteromultimerizes TRPC channels to determine their function as store-operated channels. Nat Cell Biol 9: 636-645, 2007.

18. Sydorenko V, Shuba Y and Thebault S, et al: Receptor-coupled, DAG-gated $\mathrm{Ca}^{2+}$-permeable cationic channels in $\mathrm{LNCaP}$ human prostate cancer epithelial cells. J Physiol 548: 823-836, 2003.

19. Vanden AF, Shuba Y and Roudbaraki M, et al: Store-operated $\mathrm{Ca}^{2+}$ channels in prostate cancer epithelial cells: function, regulation, and role in carcinogenesis. Cell Calcium 33: 357-373, 2003

20. El Hiani Y, Ahidouch A, Roudbaraki M, Guenin S, Brule G and Ouadid-Ahidouch $\mathrm{H}$ : Calcium-sensing receptor stimulation induces nonselective cation channel activation in breast cancer cells. J Membr Biol 211: 127-137, 2006.

21. Monteith GR, McAndrew D, Faddy HM and Roberts-Thomson SJ: Calcium and cancer: targeting $\mathrm{Ca}^{2+}$ transport. Nat Rev Cancer 7: 519-530, 2007.

22. Li Y, Jia YC and Cui K, et al: Essential role of TRPC channels in the guidance of nerve growth cones by brain-derived neurotrophic factor. Nature 434: 894-898, 2005.

23. Kindzelskii AL and Petty HR: Ion channel clustering enhances weak electric field detection by neutrophils: apparent roles of SKF96365-sensitive cation channels and myeloperoxidase trafficking in cellular responses. Eur Biophys J 35: 1-26, 2005.

24. Fabian A, Fortmann T and Dieterich P, et al: TRPC1 channels regulate directionality of migrating cells. Pflugers Arch 457: 475-484, 2008

25. Amuthan G, Biswas G, Ananadatheerthavarada HK, Vijayasarathy C, Shephard HM and Avadhani NG: Mitochondrial stress-induced calcium signaling, phenotypic changes and invasive behavior in human lung carcinoma A549 cells. Oncogene 21: 7839-7849, 2002. 
26. Huang JB, Kindzelskii AL, Clark AJ and Petty HR: Identification of channels promoting calcium spikes and waves in HT1080 tumor cells: their apparent roles in cell motility and invasion. Cancer Res 64: 2482-2489, 2004.

27. Liao J, Schneider A, Datta NS and McCauley LK: Extracellular calcium as a candidate mediator of prostate cancer skeletal metastasis. Cancer Res 66: 9065-9073, 2006.

28. Itoh T, Tanioka M, Yoshida H, Yoshioka T, Nishimoto $\mathrm{H}$ and Itohara S: Reduced angiogenesis and tumor progression in gelatinase A-deficient mice. Cancer Res 58: 1048-1051, 1998.

29. Itoh T, Tanioka $M$ and Matsuda $H$, et al: Experimental metastasis is suppressed in MMP-9-deficient mice. Clin Exp Metastasis 17: 177-181, 1999.

30. Littlepage LE, Sternlicht MD and Rougier N, et al: Matrix metalloproteinases contribute distinct roles in neuroendocrine prostate carcinogenesis, metastasis, and angiogenesis progression. Cancer Res 70: 2224-2234, 2010.
31. Wong TS, Kwong DL, Sham JS, Wei WI, Kwong YL and Yuen AP: Clinicopathologic significance of plasma matrix metalloproteinase-2 and -9 levels in patients with undifferentiated nasopharyngeal carcinoma. Eur J Surg Oncol 30: 560-564, 2004.

32. Liu Z, Li L and Yang Z, et al: Increased expression of MMP9 is correlated with poor prognosis of nasopharyngeal carcinoma. BMC Cancer 10: 270, 2010

33. Togashi $\mathrm{K}$, Inada $\mathrm{H}$ and Tominaga $\mathrm{M}$ : Inhibition of the transient receptor potential cation channel TRPM2 by 2-aminoethoxydiphenyl borate (2-APB). Br J Pharmacol 153: 1324-1330, 2008.

34. Xu SZ, Zeng F, Boulay G, Grimm C, Harteneck C and Beech DJ: Block of TRPC5 channels by 2-aminoethoxydiphenyl borate: a differential, extracellular and voltage-dependent effect. Br J Pharmacol 145: 405-414, 2005. 